Supporting Information

\title{
Formation of Isolated Pseudo-Polyrotaxane Nanosheet Consisting of $\alpha$-Cyclodextrin and Poly(ethylene glycol)
}

Shuntaro Uenuma, Rina Maeda*, Hideaki Yokoyama and Kohzo Ito*

Department of Advanced Materials Science, Graduate School of Frontier Sciences, The University of Tokyo, Kashiwa-city, Chiba 277-8561, Japan

[1] WAXS profiles of PPR consisting of the PEG with a variety of molecular weight. .2

[2] Fitting a nanosheet form factor to the SAXS profiles of PPR2k, PPR3.4k, PPR4k, PPR4.6k, and PPR6k.

[3] SAXS profiles of PPR400, PPR600, PPR10k, PPR12k, PPR20k, and PPR35k ....................4

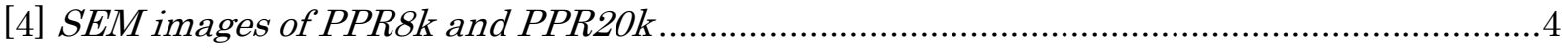

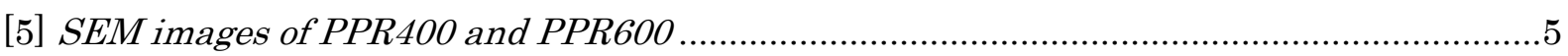

[6] WAXS profiles of PPR consisting of the end functionalized PEGS .......................................5

[7] Fitting a nanosheet form factor to the SAXS profiles of iBuPPR2k, PiPPR2k, $\mathrm{NH}_{2} P P R 2 k$,

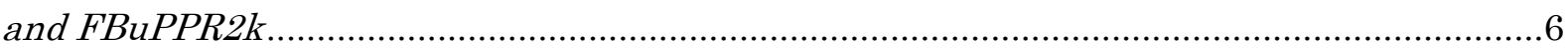

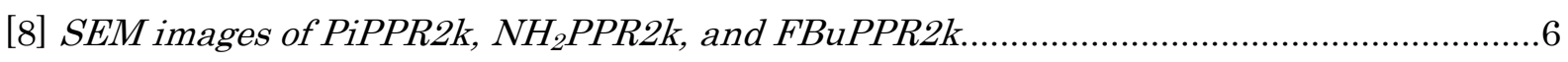

[9] WAXS profiles of PPR1.25k-4, PPR2.50k-4, and PPR1.25k-8...........................................

[10] Fitting a nanosheet form factor to the SAXS profiles of PPR1.25k-4, PPR2.50k-4, and

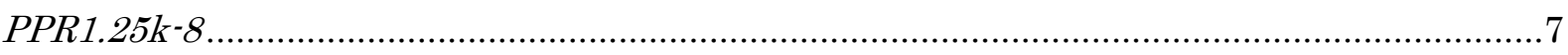

[11] SEM images of PPR1.25k-4, PPR2.50k-4, and PPR1.25k-8...........................................

[12] Characterization of the end functionalized PEG .........................................................

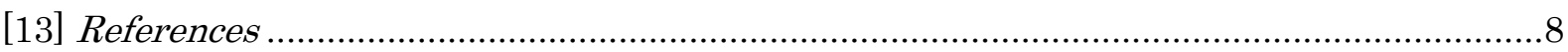


[1] WAXS profiles of PPR consisting of the PEG with a variety of molecular weight

The WAXS profiles of water dispersions of PPR400, PPR600, PPR1k, PPR2k, PPR3.4k, PPR4k, PPR4.6k, PPR6k, PPR8k, PPR10k, PPR12k, PPR20k, and PPR35k are shown in Figure S1. The characteristic peaks based on the conventional hexagonal columnar structure are marked.

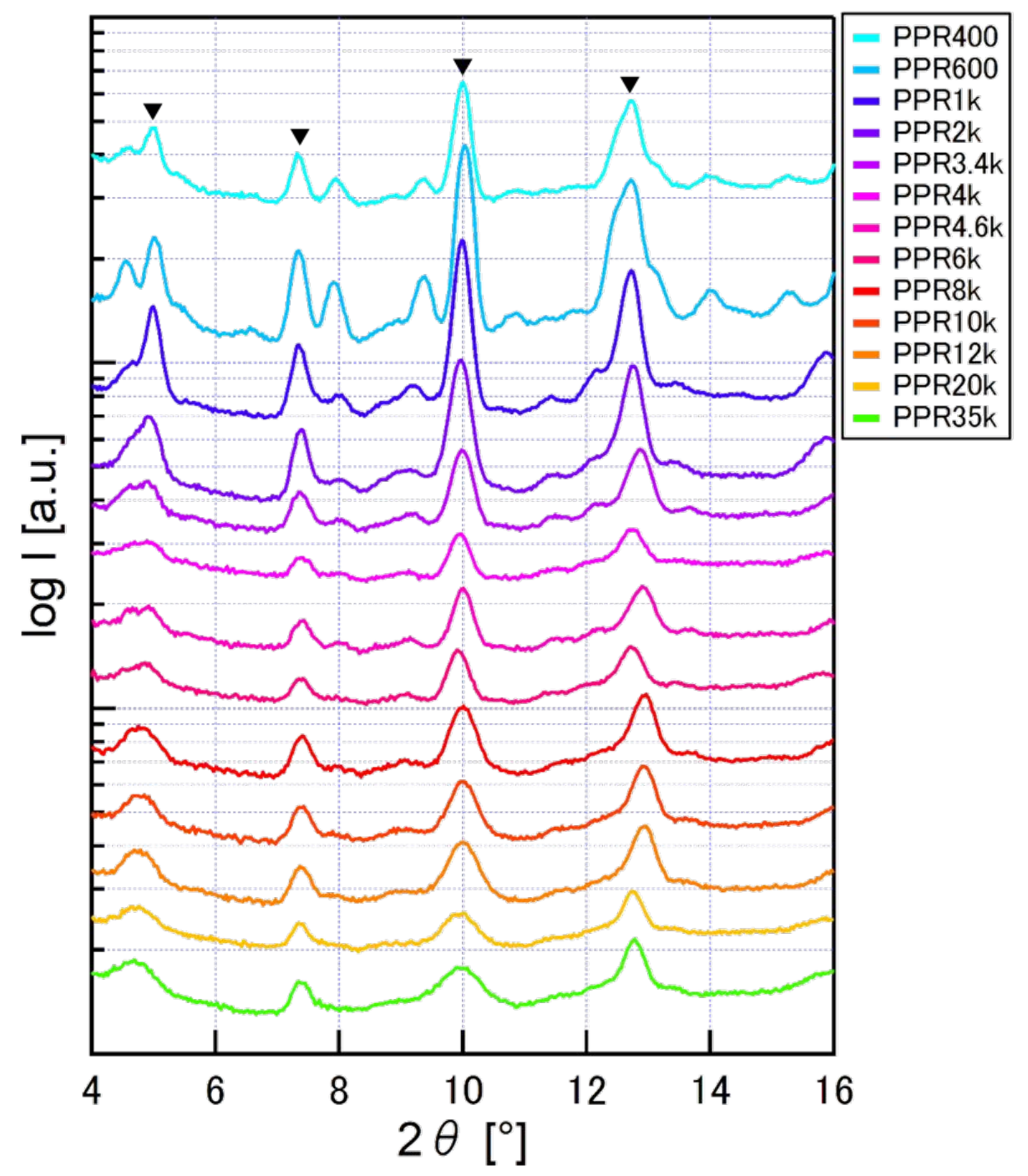

Figure S1. The WAXS profiles of PPR400, PPR600, PPR1k, PPR2k, PPR3.4k, PPR4k, PPR4.6k, PPR6k, PPR8k, PPR10k, PPR12k, PPR20k, and PPR35k. 
[2] Fitting a nanosheet form factor to the SAXS profiles of PPR2k, PPR3.4k, PPR4k, PPR4.6k, and PPR6k

The SAXS profiles of water dispersions of PPR2k, PPR3.4k, PPR4k, PPR4.6k, and PPR6k are shown in Figure S2. The graph of nanosheet form factor ${ }^{1}$ are overlaid in each figure.

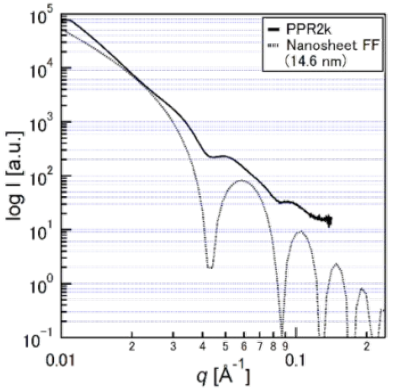

(a)

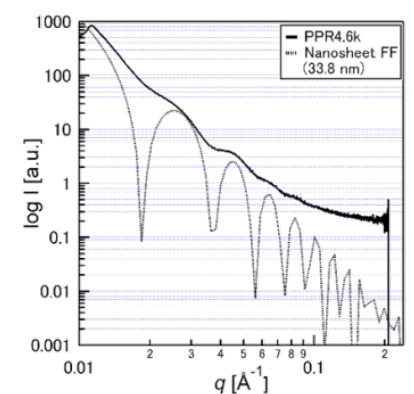

(d)

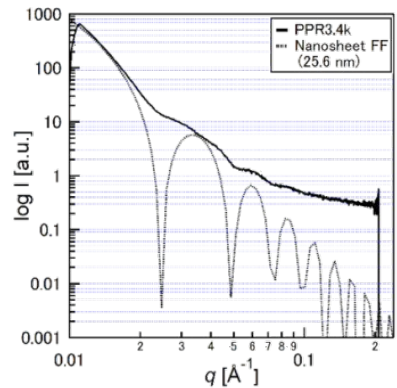

(b)

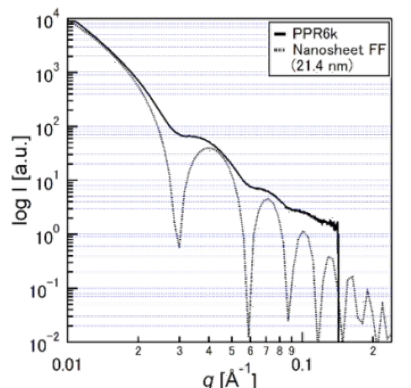

(e)

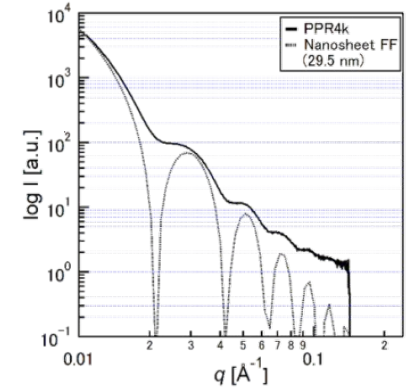

(c)

Figure S2. The SAXS profiles of water dispersion of (a) PPR2k, (b) PPR3.4k, (c) PPR4k, (d) PPR4.6k, and (e) PPR6k and the fitted nanosheet form factors are overlaid. The thicknesses of nanosheets form factors are written in the graph legends. 
[3] SAXS profiles of PPR400, PPR600, PPR10k, PPR12k, PPR20k, and PPR35k

The SAXS profiles of water dispersions of PPR400, PPR600, PPR10k, PPR12k, PPR20k, and PPR35k are shown in Figure S3.

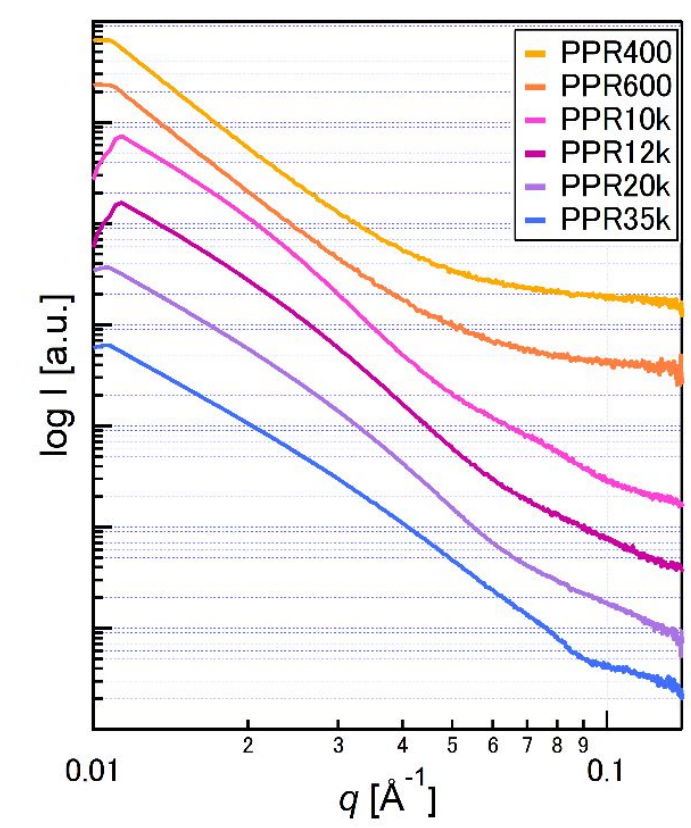

Figure S3. The SAXS profiles of PPR400, PPR600, PPR10k, PPR12k, PPR20k, and PPR35k.

\section{[4] SEM images of PPR8k and PPR2Ok}

The SEM images of PPR8k and PPR20k are shown in Figure S4.

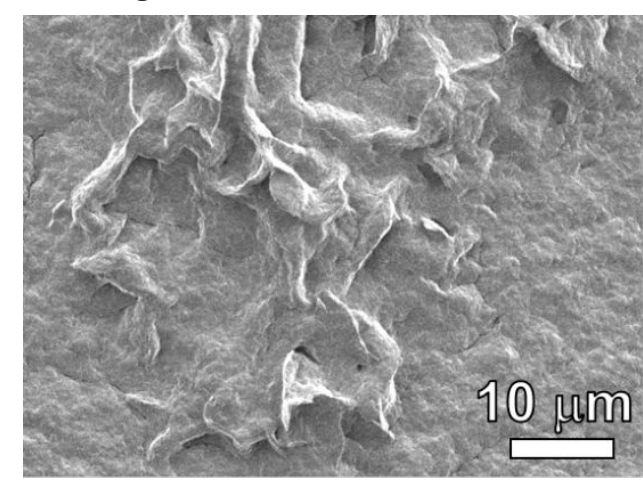

(a)

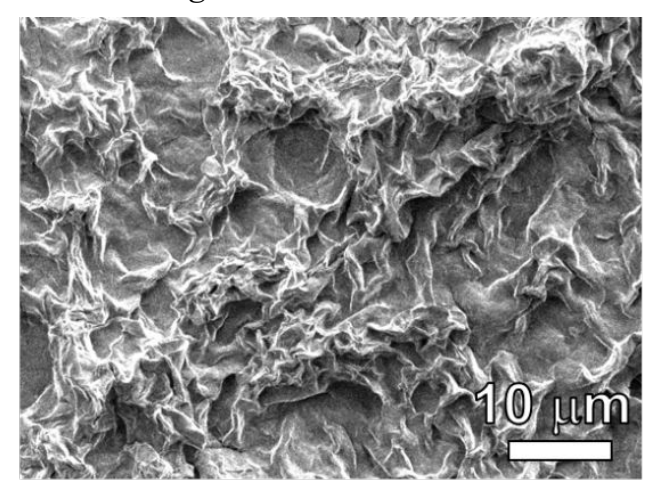

(b)

Figure S4. The SEM images of (a) PPR8k and (b) PPR20k. 
[5] SEM images of PPR400 and PPR600

The SEM images of PPR400 and PPR600 are shown in Figure S5.

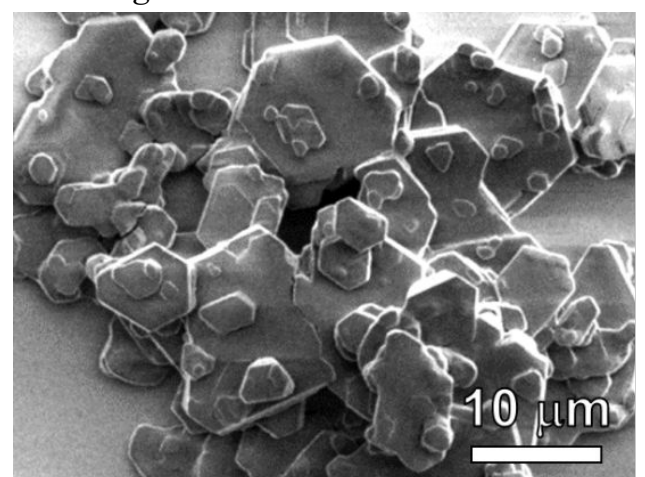

(a)

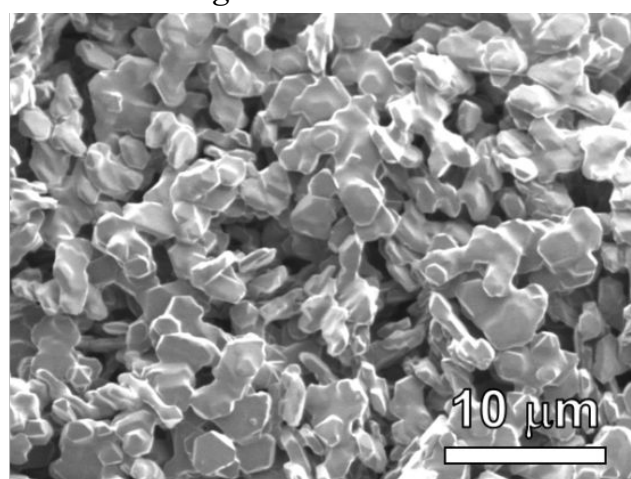

(b)

Figure S5. The SEM images of (a) PPR400 and (b) PPR600.

[6] WAXS profiles of PPR consisting of the end functionalized PEGS

The WAXS profiles of $n$ PePPR $2 \mathrm{k}, n$ BuPPR2k, PrPPR $2 \mathrm{k}, i$ BuPPR2k, PiPPR $2 \mathrm{k}, \mathrm{NH}_{2} \mathrm{PPR} 2 \mathrm{k}$, and FBuPPR2k are shown in Figure S6. The characteristic peaks derived from the conventional hexagonal columnar crystal are marked.

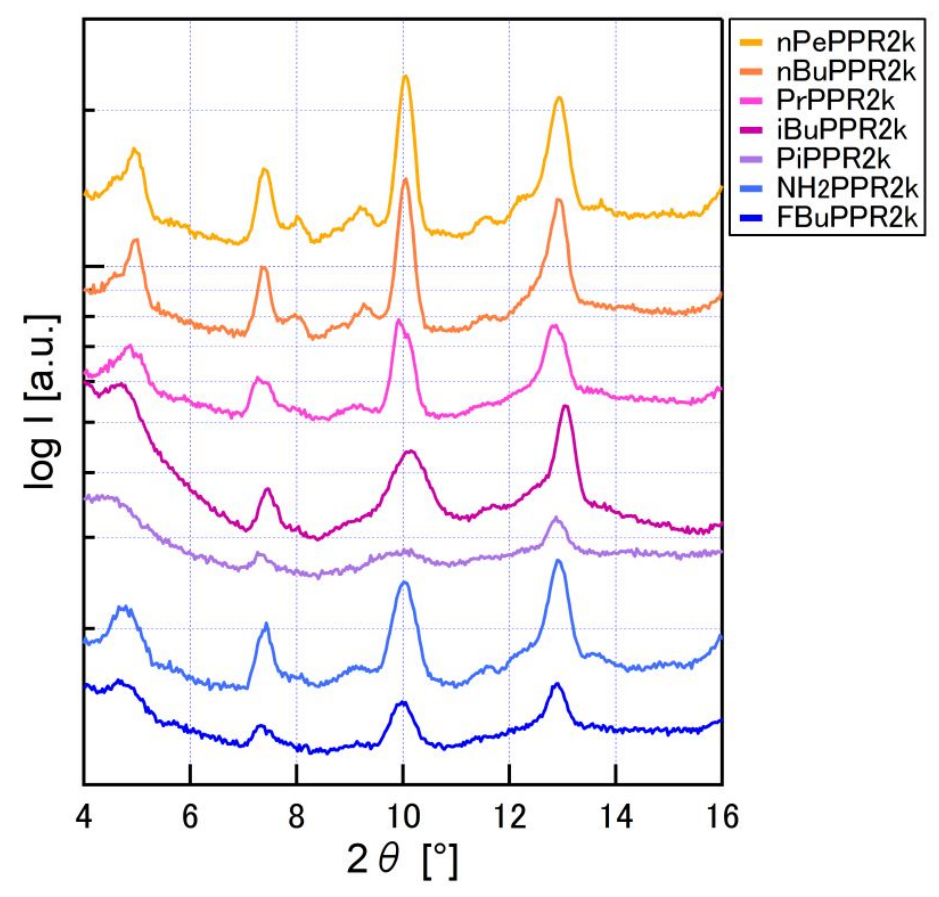

Figure S6. The WAXS profiles of $n$ PePPR2k, $n$ BuPPR2k, PrPPR2k, $i$ BuPPR2k, PiPPR2k, $\mathrm{NH}_{2} \mathrm{PPR} 2 \mathrm{k}$, and FBuPPR2k. 
[7] Fitting a nanosheet form factor to the SAXS profiles of iBuPPR2k, PiPPR2k, $\mathrm{NH}_{2} P P R 2 k$, and FBuPPR2k

The SAXS profiles of water dispersion of $i$ BuPPR2k, PiPPR2k, $\mathrm{NH}_{2} \mathrm{PPR} 2 \mathrm{k}$, and FBuPPR2k are shown in Figure S7 and the graph of nanosheet form factor ${ }^{1}$ are overlaid in each figure.

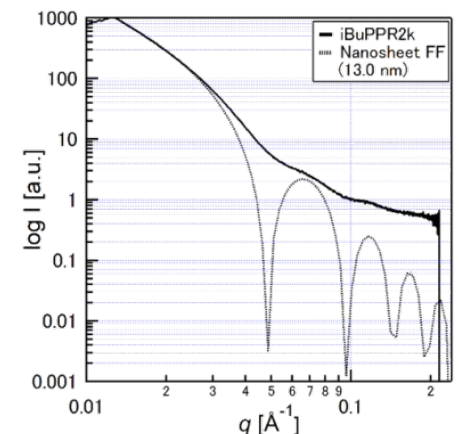

(a)

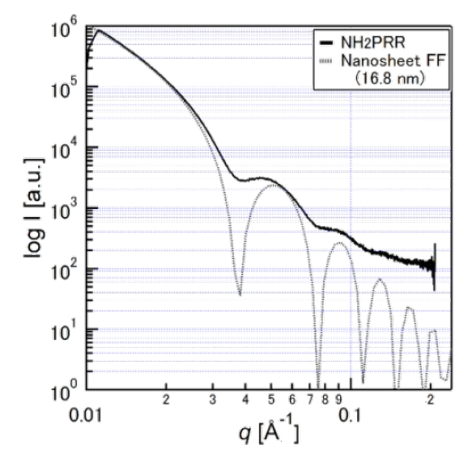

(c)

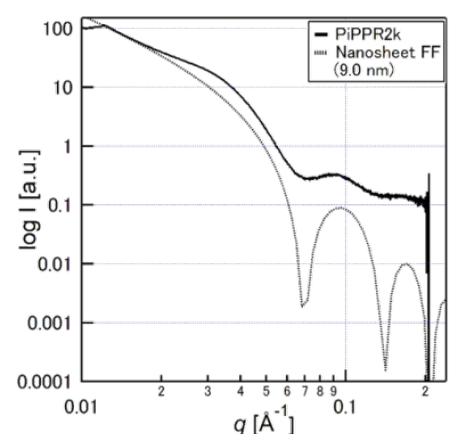

(b)

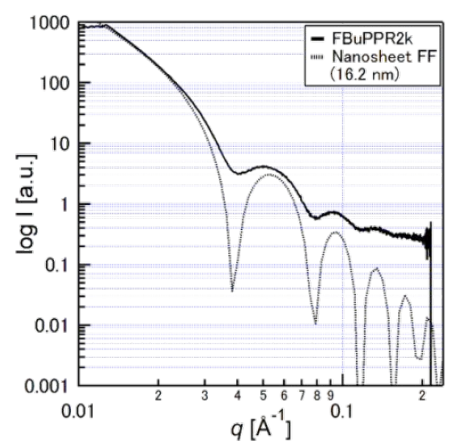

(d)

Figure S7. The SAXS profiles of water dispersion of (a) iBuPPR2k, (b) PiPPR2k, (c) $\mathrm{NH}_{2} \mathrm{PPR} 2 \mathrm{k}$, and (d) FBuPPR2k and the fitted nanosheet form factors are overlaid. The thicknesses of nanosheets form factors are written in the graph legends.

[8] SEM images of PiPPR2k, $\mathrm{NH}_{2} P P R 2 k$, and FBuPPR2k

The SEM images of PiPPR2k, $\mathrm{NH}_{2} \mathrm{PPR} 2 \mathrm{k}$, and FBuPPR2k are shown in Figure S8.

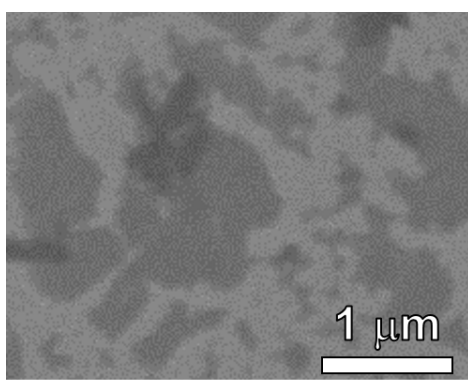

(a)

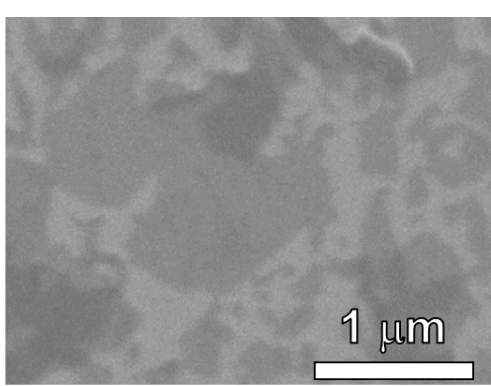

(b)

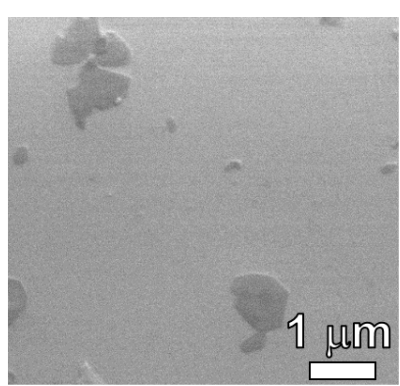

(c)

Figure S8. The SEM images of (a) PiPPR2k, (b) $\mathrm{NH}_{2} \mathrm{PPR} 2 \mathrm{k}$, and (c) FBuPPR2k. 
[9] WAXS profiles of PPR1.25k-4, PPR2.50k-4, and PPR1.25k-8

The WAXS profiles of PPR1.25k-4, PPR2.50k-4, and PPR1.25k-8 are shown in Figure S9. The characteristic peaks based on the conventional hexagonal columnar crystal are marked.

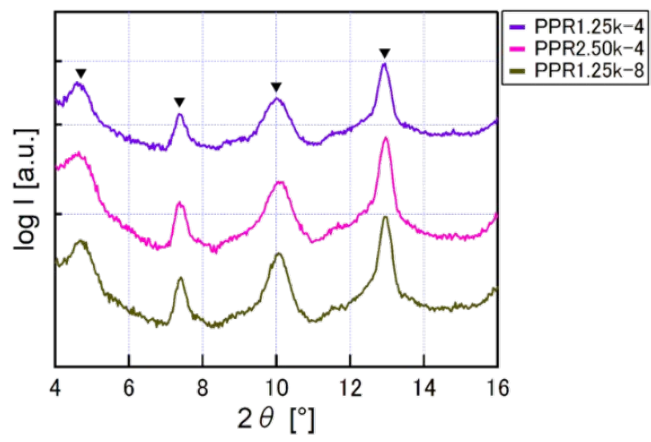

Figure S9. The WAXS profiles of PPR1.25k-4, PPR2.50k-4, and PPR1.25k-8.

[10] Fitting a nanosheet form factor to the SAXS profiles of PPR1.25k-4, PPR2.50k-4, and PPR1.25k-8

The SAXS profiles of water dispersion of PPR $1.25 \mathrm{k}^{-} 4$, PPR2.50k-4, and PPR $1.25 \mathrm{k}^{-} 8$ are shown in Figure S10 and the graph of nanosheet form factor ${ }^{1}$ are overlaid in each figure.

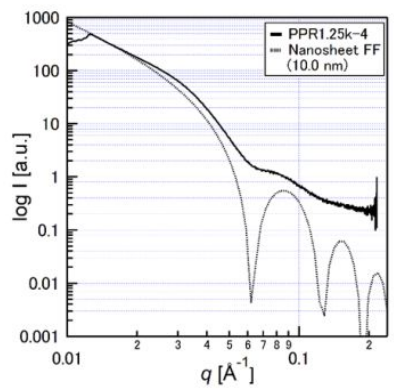

(a)

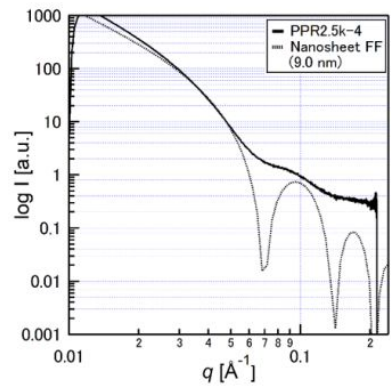

(b)

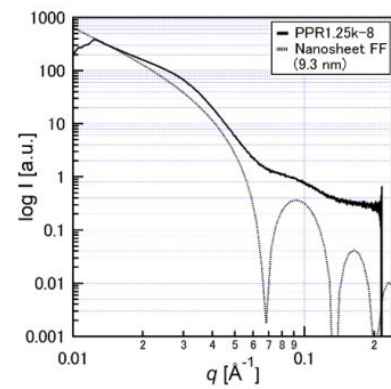

(c)

Figure S10. The SAXS profiles of water dispersion of (a) PPR1.25k-4, (b) PPR2.50k-4, and (c) PPR $1.25 \mathrm{k}-8$ and the fitted nanosheet form factors are overlaid. The thicknesses of nanosheets form factors are written in the graph legends.

\section{[11] SEM images of PPR1.25k-4, PPR2.50k-4, and PPR1.25k-8}

The SEM images of PPR1.25k-4, PPR2.50k-4, and PPR1.25k-8 are shown in Figure S11.

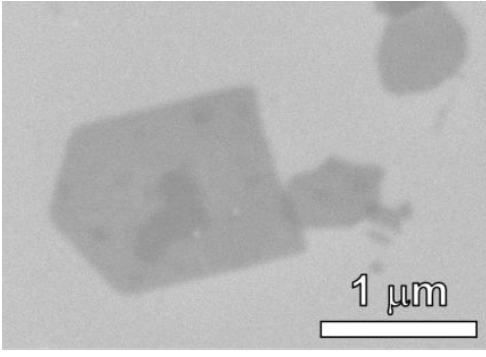

(a)

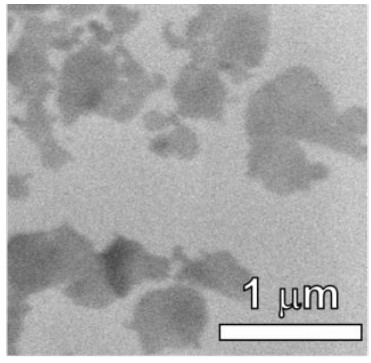

(b)

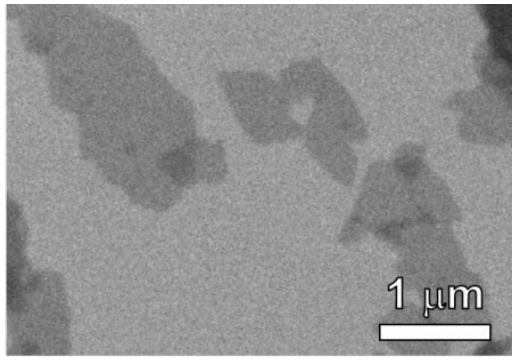

(c)

Figure S11. The SEM images of (a) PPR1.25k-4, (b) PPR2.50k-4, and (c) PPR1.25k-8. 


\section{[12] Characterization of the end functionalized PEG}

${ }^{1} \mathrm{H}-\mathrm{NMR}$ spectra of PrPEG2k, $n$ BuPEG2k, $n$ PePEG, ${ }_{\imath}$ BuPEG2k, PiPEG2k, and FBuPEG2k are shown in Figure S12. The assignments of the peaks to the chemical structures are embedded in each figure. The modification ratios of end hydroxyl groups of PrPEG2k, $n$ BuPEG2k, $n$ PePEG, $i$ BuPEG2k, PiPEG2k, and FBuPEG2k could be calculated to be $98 \%$, $93 \%, 97 \%, 94 \%, 62 \%$, and $88 \%$, respectively, using the peaks at $4.2-4.5 \mathrm{ppm}\left(-\mathrm{CH}_{2}-\mathrm{OCO}^{-}\right)$ and $3.4-3.8 \mathrm{ppm}\left(\mathrm{CH}_{2}-\mathrm{CH}_{2}{ }^{-} \mathrm{O}^{-}\right)$.

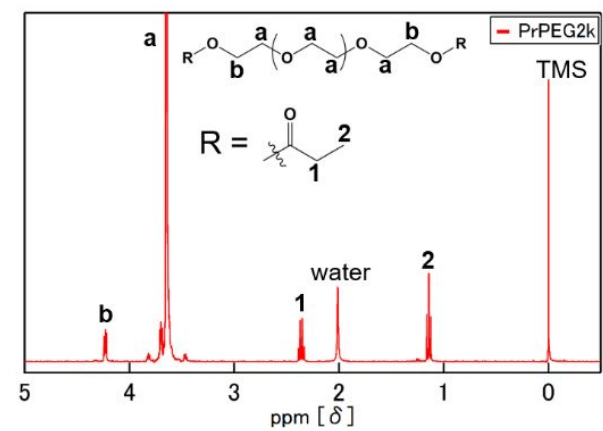

(a)

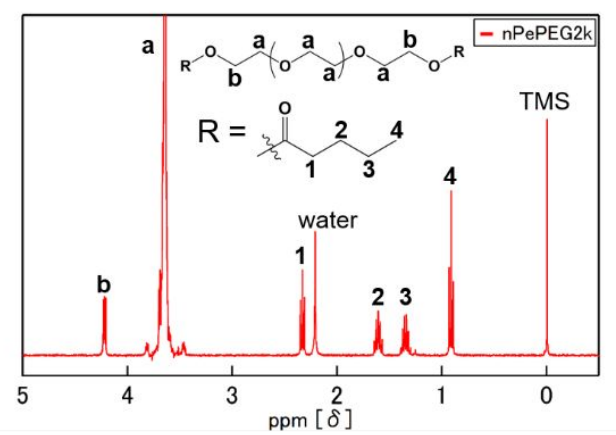

(c)

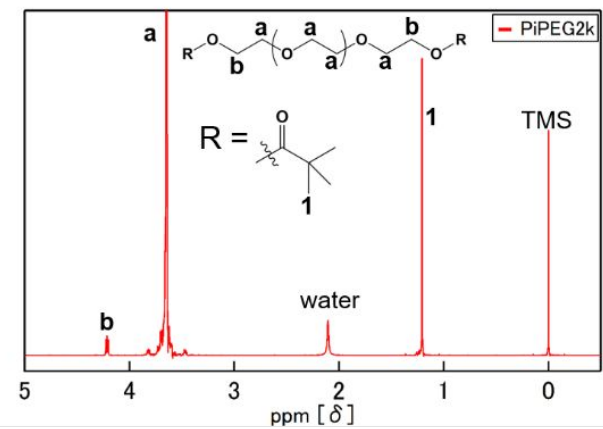

(e)

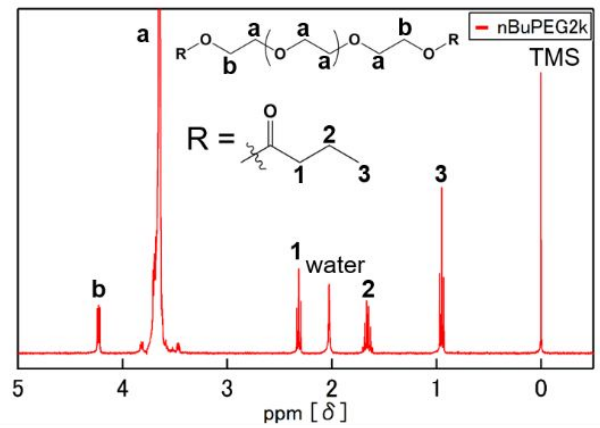

(b)

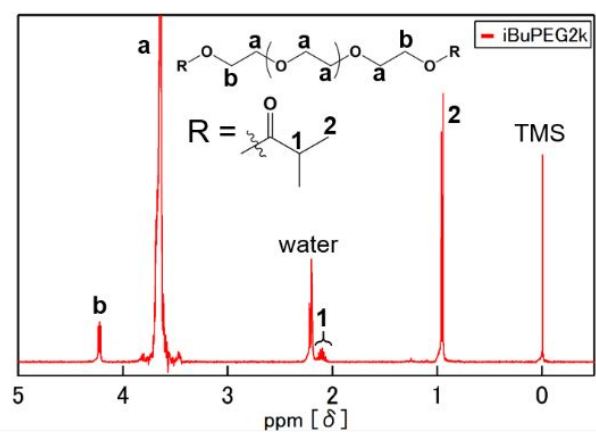

(d)

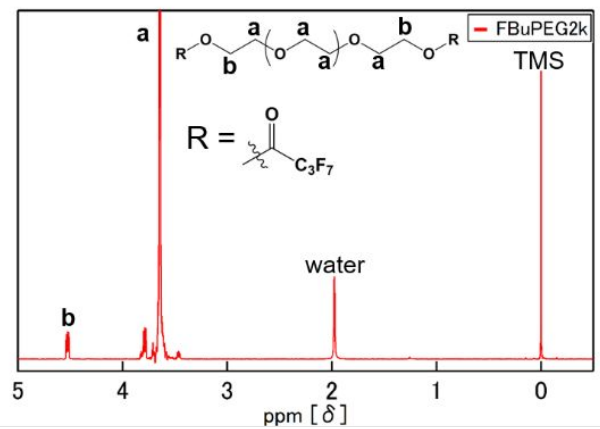

(f)

Figure S12. ${ }^{1} \mathrm{H}-\mathrm{NMR}$ spectra of (a) PrPEG2k (b) $n$ BuPEG2k, (c) $n$ PePEG, (d) ${ }_{2}$ BuPEG2k, (e) PiPEG2k, and (f) FBuPEG2k.

\section{[13] References}

(1) Miyamoto, N.; Nakato, T. Liquid Crystalline Inorganic Nanosheet Colloids Derived From Layered Materials. Isr. J. Chem. 2012, 52(10), 881-894. 